\title{
MENINGITIS TUBERCULOSA Y SÜ TRATAMIENTO CON ESTREPTOMICINA
}

\author{
(Resumen de los datos obtenidos en 107 niños hospitalizados \\ en el Hospital Luis Calvo Mackenna).
}

\author{
Por los Dres. JORGE E. HOWARD. RAUL MATTE L., MARIANo LATORRE. \\ y ALFREDO VARGAS BAEZA \\ Haspital L. Calvo Mzckenna. Cáredra de Pidiatria dat Prof. A. Ariztía.
}

I) Generalidades: El presente trabajo se basa en 107 niños con meningitis tiberculosa hospitalizados en el Hospital Luis Calvo Mackenna en el período comprendido entre junio de 1947 a septiembre de 1950. Todos estos niños han sido seguidos, ya sea directamente, o por medio de cuestionarios si en la actualidad viven fuera de Santiago, hasta el mes de notiembre de 1951 . Es así que el periodo de observación mínimo de los enfermos actualmente vivos es de 14 meses a partir de la iniciación de su cuadro clinico. Para estudiar los efectos del tratamiento se han eliminado todos aquellos casos que fallecieron durante la primera semana de hospitalización. En estas condiciones nuestros resultados son los siguientes: de 107 casos tratados, sobreviven 41 , o sea, un $37.9 \%$; fallecieron en el hospital 58 enfermos y 8 presumimos que han fallecido, ya que fueron retirados muy graves o en estado agónico, lo que da un total de $62.1 \%$ de mortalidad.

En lo referente al tratamiento se han seguido dos planes principales: el tratamiento continuo y el tratamiento discontinuo. Llamamos tratamiento continuo aquél en que se ha usado la vía intramuscular diariamente por espacio de 5 meses como mínimo e inyecciones intratecales diariamente durante 15 a 30 días, para luego continuar día por medio, bisemanalmente, y finalmente, una vez a ia semana, hasta la normalización del líquido céfaloraquideo, o sea, un plazo no inferior a 9 meses. Llamamos tratamiento discontinuo aquél en que las inyecciones intramusculares diarias se colocaron por períodos menores de 5 meses, o bien, hubo lapsos en que se interrumpió el tratamiento intramuscular o el intratecal. En ambos planes de tratamiento las dosis usadas fueron de 30 a 40 miligramos por kilo de peso y por día, por la vía intramuscular y de 2 a 3 miligramos por día y por kilo de peso por vía intratecal.

Para tratar de probar las bondades de uno u otro método, se tomó el tercer mes como punto básico, descartando así todos los casos fallecidos antes de esta época y reuniendo en dos grupos los que a. esa fecha (90 días) estaban vivos, aunque después fallecieron.

En esta forma tuvimos 36 casos tratados con el método continuo, de Jos cuales posteriormente fallecieron 10 y 27 casos con el tratamiento discontinuo, de los cuales fallecieron posteriormente 12. Los porcentajes de mortalidad son entorices de $27 \%$ para el tratamiento continuo contra un $44 \%$ para el tratamiento discontinuo, lo que nos indica las ventajas del método continuo. Además, de los 36 enfermos sometidos al tratamiento continuo sólo uno tuvo una recaida, en tanto que entre los 27 del plan disconti- 
nuo, 11 tuvieron recaídas. De los 12 que tuvieron recaídas, murieron 8 .

II) Diversos factores que tienen importancia en el resultado del tratamiento.

a) Edad: Hemos dividido a los niños en tres grupos para estudiar la importancia del factor edad: menores de 2 años, de 2 a 6 años y mayores de 7 años, 0 sea, lactantes, pre-escolares y escolares. Entre los lactantes, de 26 casos murieron 18 , o sea, un $69 \%$; en pre-escolares, de 38 casos murieron 22 , o sea, un $57 \%$ y entre 43 escolares murieron 26 , o sea, un $60 \%$. Por lo tanto, vemos que la mortalidad es mayor entre los lactantes y sensiblemente igual en los pre-escolares y escolares.

b) Tiempo transcurrido entre el comienzo aparente de los sintomas y la iniciación del tratamiento: Para estudiar este factor hemos agrupado el material en 3 grupos: menor de 3 dias, de 3 a 6 días y más de 7 días. En el primer grupo, o sea, en aquéllos en que el tratamiento se inició antes de los 3 días de haber comenzado los síntomas, de 15 casos murieron 10, o sea, el $66 \%$; en el segundo grupo, de 26 casos murieron 16, el $61 \%$, y en el tercer grupo, de más de 7 dias de sintomatologia, de 66 casos murieron 40 , o sea, el $60 \%$. Vemos aquí que no influye en la sobrevida el lapso que media entre el comienzo de la enfermedad y el comienzo del tratamiento. Además, vemos que la mayor parte de los casos que ingresan al hospital lo hacen después de una semana de iniciada la sintomatología.

c) Estreptomicina anterior: De 17 enfermos que estaban recibiendo o acababan de recibir estreptomicina por alguna otra lesión tuberculosa, murieron 12, sea, un $70 \%$, lo que indica que el haber recibido o estar en el curso de un tratamiento estreptomicínico agrava el pronóstico de la meningitis tuberculosa.

d) Lesión tuberculosa pulmonar concomitante: En nuestro material de 107 enfermos, 50 , o sea, el $55.1 \%$, tenían un complejo primario en evolución; 41, o sea, el $38.3 \%$, tenían una tuberculosis miliar, $y$ en 7 enfermos, o sea, el $6.6 \%$, no se encontró lesión tuberculosa pulmonar o ésta estaba calcificada. En estos últimos 7 casos, el diagnóstico de meningitis tuberculosa se comprobó a la autopsia en 5. En el grupo con complejo primario fallecieron 32 , o sea, el $54 \%$; en el segundo grupo, con tuberculosis miliar, fallecieron 29 , o sea, el $70 \%$. Se deduce, por lo tanto, que el haber una tuberculosis miliar pulmonar concomitante ensombrece el pronóstico.

III) Mortalidad: De 107 enfermos fallecieron 58 , o sea, el $62.1 \%$. Como se ha observado en otras partes, en nuestro material también hemos encontrado una mayor mortalidad en los dos primeros meses, $67 \%$. Después del octavo mes las muertes son la excepción.

IV) Complicaciones del tratamiento: En el curso de la meningitis tuberculosa tratada con estreptomicina, dos son las grandes complicaciones del tratamiento: la hidrocefalia y la hemiplegia. Hemos estudiado el síndrome hidrocefálico en 25 niños y hemos podido determinar los siguientes hechos: puede aparecer en cualquiera época en el curso de la enfermedad; existen dos mecanismos de producción: por hipersecreción de los plexos coroídeos y por falta de reabsorción debido a la presencia de exudados en las cisternas basales. Este segundo mecanismo es más corriente y más grave. Es importante determinar el tipo de hidrocefalia, lo que se hace por la neumoencefalografia, ya que algunos casos pueden ser mejorados por medios quirúrgicos.

Hemos estudiado 12 enfermos con hemiplegia, la cual es siempre completa y aparece en cualquier período de la enfermedad. Su mecanismo de producción sería por exudados fibrinosos perivasculares que obstruyen el vaso. Para localizar el sitio de la hemiplegia se recurre al electroencéfalograma y a las arteriografías. La mayoría de los enfermos con hemiplegia sobreviven. 
V) Control posterior de los enfermos: El tiempo de sobrevida, o sea, hasta la última vez que obtuvimos informaciones - que examinamos personalmente a los enfermos, ha sido el siguiente: con sobrevida de $4 \frac{1 / 2}{2}$ años: 2 enfermos; con sobrevida de $3 \frac{1}{2}$ años: 10 enfermos; con sobrevida de $2 \frac{1}{2}$ años: 14 enfermos y con sobrevida de menos de $2 \frac{1}{2}$ y más de 14 meses: 15 enfermos.

Entre los 41 sobrevivientes actualmente tenemos 25 niños que hemos catalogado como sanos, o sea, el $60 \%$ de los que viven y el $23 \%$ del total de niños con meningitis tuberculosa.

En los 16 enfermos que sobreviven, pero que tienen secuelas, hemos hecho los siguientes diagnósticos: hemiplegia en 7 , marcha titubeante en 3 , crisis de violencia en 3 , gran disminución de la visión en 2, gran hidrocefalia en 2, epilepsia jacksoniana en 1, cleptomanía en 1, sordera en 1.

\section{Summary.}

The authors summarize the data obtained from 107 children with tuberculous meningitis treated with streptomycyn, studied at the Calvo Mackenna Hospital. Those that recovered have been studied for a minimum period of 14 months. Of the 107 children, $38 \%$ are alive.

From their study they conclude that:

1) The continual method of treatment, i. e.: daily intramuscular streptomycin injections for a period not less than 5 months, daily intraspinal injections for 15 to 30 days, followed by every other day spinal streptomycin for several months and then for a weakly injection until normalization of the cerebrospinal fluid, is superior to other forms of treatment.

2) Mortality is greater in the smaller infants.

3) There is no corelation between survival and precocity of treatment.

4) Previous streptomycin therapy and concomitant miliary lung tuberculosis are bad prognostic signs.

5. There are two major complications during treatment: hydrocephalus and hemiplegia.

6) The survival periods were the following: $4 \frac{1}{2}$ years, 2 children; $3 \frac{1 / 2}{2}$ years, 10 children; $2 \frac{1}{2}$ years, 14 children and less than $2 \frac{1}{2}$ years but more than 14 months, 15 children.

7) Among those that are alive $60 \%$ have no secuelae. 